\title{
Ontology-based User Model for Personalized Search in a Social Network
}

\author{
Maha Maalej $^{2}$, Achraf Mtibaa ${ }^{1}$, Faïez Gargouri ${ }^{2}$ \\ ${ }^{1}$ National School of Electronic and Telecommunications of Sfax, Tunisia \\ ${ }^{2}$ Higher Institute of Computer Science and Multimedia of Sfax, Tunisia \\ \{maha.maalej, achrafmtibaa, faiez.gargouri\}@gmail.com
}

\begin{abstract}
Nowadays, internet users are faced with a big quantity of information on the Web. This leads to a difficulty in obtaining information relevant to their needs and adapted to their contexts. In order to better solve these problems, personalized systems where a user model contains information about user context and profile have arisen. As a means to represent user information, ontology constitutes a better technique of Semantic Web that improves the way to maintain user information. In this paper, we propose an approach through that we construct the ontological user model in a first time and we integrate this model in a personalized system in a second time. In this context, we present main concepts of our ontology. We have enriched our ontology with SWRL rules on the one hand and FOAF ontology concepts on the other hand. This has the advantage of deducing services adapted to the user through a personalized system. After that, we expose functionalities of our system with evaluation.
\end{abstract}

Keywords: inference, SWRL rules, user model ontology, personalization, handicraft domain.

\section{Introduction}

The arrival of the social web has brought new opportunities to access and share knowledge. However, many difficulties related to the information and communication technologies (ICT) can face many people and prevent them from exploiting their benefits. Social Network Services (SNS) wikis and blogs are examples of social web applications that generate a large amount of information and, therefore, require specific techniques so as to apprehend appropriate information to users. When SNS impose challenges on information access, they may also have a dominant role in influencing ICT use. In developing countries, some people can be considered as experienced ICT users and some of them can effectively use these technologies across the web.

The main contribution of this paper is to show how to produce ontology-based search mechanisms that enable users in the process of accessing information using keywords.

With the goal of optimizing search results, researchers in the semantic field have incorporated techniques with a variety of other research areas, and implemented a 
number of practical systems. Search mechanisms with semantic features require that the mechanism be based on the model of domain knowledge. This means that knowledge can be better represented by ontologies. These ontologies are Semantic Web technologies which are often written in OWL (Ontology Web Language) language.

This work is involved in the context of the project BWEC (Business for Women of Emerging Country) that aims to improve the socio-economic situation of handicraft women. The project treats handicraft women from Tunisia and Algeria. In this context, an interactive system is going to be built based on many works and steps. Our work is focused mainly on the first step which is the user modeling that concerns the handicraft woman profile in our case. To do that, we have made interviews to collect information about these women and their productions. These interviews cover five main topics: craft production nature, production process, the use of coordination tools, the latent needs and socio-demographic data. After analyzing these interviews, we noticed that handicraft women can have multiple needs by interacting with a personalization system. Indeed, she may request raw material with a suitable price. She can also look for a supplier that is nearby her house. She may, as well, look for a particular training to improve her skills. Several sides of handicraft women must be specified in the user model that we try to accomplish such as production, sale, knowledge and skills, using new technologies, training, etc. To treat these sides, the user model must be extensive and expandable. It contains concepts that are related to the user's personal side that represents the user profile such as age, name, level of education, intellectual level, marital status, etc. It contains other concepts that are related to the handicraft field. It contains as well another type of concepts representing contextual information such as location.

This paper proposes an ontology-based approach that aims to provide a solution to get semantic search results corresponding to many users at the same time while respecting the individuality of each one.

In the rest of this paper, we present the motivations of our proposed solution, in section 2 . In section 3 , we present the basic notions related to our work. Then, we provide our ontology for user model and for handicraft domain, in section 4 . In section 5, we present our system to exploit the ontology for searching on the web, searching on Facebook social network and for local search in the ontology. In section 6 , we give experimentation results with discussion. We conclude by giving a conclusion and presenting future works in section 7 .

\section{Motivations}

As ICT technologies emerge, they become considerably used by people so as to access information through the internet. However, not everyone can use these techniques due to many reasons: internet is not covered in rural areas, or people do not have idea about using such technologies. For this reason, there is a need to an enhanced search application that can be used by these people. Thus, they will be able to access information although the lack of knowledge about these advanced technologies. 
We notice that in developing countries such as Tunisia and Algeria there is a big number of people who use ICT technologies and access internet. However, this is not the case in all areas of the countries. In fact, the rural areas suffer from the lack of use of these technologies, it can be either technological, educational or economic difficulties that restrain them.

After analyzing the interviews, we find that $12.85 \%$ of interviewed handicraft people are illiterate and $40 \%$ have secondary level. $91.25 \%$ of people are fine with using ICT technologies, while $8.75 \%$ are not ready to use them. $43 \%$ of people who are ready to use ICT technologies belong to the interval of age $[30,40]$ and $29 \%$ are between 40 and 50 years old.

Social networking is a known concept that exists from a long time ago. However, with technological progress and Internet advances, social network became a tool that connects people and allows a new way of contact. In this context, we notice the emergence of many social network sites with the advance of Web 2.0 such as Facebook and Twitter.

With the increasing use of ICT technologies and social network site, user information is treated so as to build upon to permit personalization. In fact, social profiles contain information about social networks users. Treating such information has the aim to deliver users with personalized experience when searching for information.

Personalization in a system represents the way this system provides information relevant to a user according to his preferences, interests and context. In fact, context is an important concept in personalization. Indeed, it permits to surround the knowledge about a situation of a user when he is interacting with the system.

The proliferation and the wide use of social network sites have influenced research related to semantic web. In fact, knowledge capture through social networking opens new manners in which ontologies are developed and used. For this reason, many ontologies have emerged, facilitating the process of representing knowledge that is extracted from social network.

\section{Background and Related Works}

The term "ontology" has been known in the field of human sciences before being defined in the computer field. It appeared in metaphysics and it is interested in the notion of existence in the fundamental categories of the existent, and it studies the most general properties of being. Then, it has become used in the computer field, where it was defined, by Gruber, as "an explicit specification of a conceptualization of a given domain" [13].

Ontologies developed in OWL incorporate internal logical rules that are defined by the semantics of the language. Inference is the deduction of explicit information from implicit data. This inference can be achieved through inference rules that can be associated with the ontology and performed by the reasoner. These rules are defined by the programmer after modeling and completing the implementation of the ontology.

For the development of these rules we employed SWRL (Semantic Web Rule Language) rule language. In fact, it offers a powerful expansion technique that 
permits user-defined procedures to be exploited in rules. These procedures are called built-ins and are predicates that accept one or more arguments. A number of basic built-ins are already defined in the SWRL presentation.

Social networks offer several characteristics. In fact, users can change their profile information by adding or removing information such as contact information, photos, hobbies, books, movies, music, and more. Users can browse the profiles of other users through the search and subsequently get lists of their friends and put them into categories. A social network is a model in which social entities such as people, organizations and locations are interconnected by certain relationships. In order to visualize a social network, several techniques appeared like the sociogram [30], the graph [7], the adjacency matrix [36] and the ontology [24]. A synthesis of these different forms of social network representation is presented in our previous work [25]. Since social networks are developed by different types of relationships, it may be impossible for graphs and numerical values of matrices to explain all semantic relationships. As a solution, ontology can be adapted to represent social networks. The first ontology built to represent social networks elements is SIOC (Semantically Interrelated Online Communities) [3]. Nevertheless, the first ontology invented to represent people and relationships between them is FOAF (Friend of A Friend) [5].

Social networking sites use the users' contextual information in order to adapt their use according to user' own interests. There are several works on the literature that use context in social networks. Each of these works deals with an aspect that shows the importance of using the context in social networks. Some discuss the importance of using contextual information such as Brézillon [4] and Wang [39]. Others deal with the extraction of contextual information Zitnik [44], Ghita [10], Joly [20], Narayanan [31] and White [40]. Others deal with the use of contextual information in social networks through mobile devices like Johansson [19], Zigkolis [43], Hardy [14] and Qiao [33].

Personalization approaches are related to the way personalization is done. It is an important aspect nowadays, with the search for information requires taking into account the need of the user, and the recommendation of people or elements requires knowing the interests and preferences of the user.

Kostadinov [23] defines the personalization of information as a set of individual preferences, ranking criteria or specific semantic rules for a user or group of users. This way of definition is used to describe the interest of the user, the level of quality of the data he prefers or the way in which the data is presented. The methods used for the implementation of personalization of the contents are the result filtering, the classification of the obtained results, the recommendation, the personalized information search and the automatic filling of forms.

Personalization in Personalized Information Retrieval (PIR) systems can be accomplished by personalizing the query, personalizing search results, or both. This personalization can be achieved by a number of techniques, which are: pre-sorting results, filtering results and sorting results [29].

Contextual information retrieval (CIR) has emerged in recent years [18], with the aim of optimizing the relevance of research results. It involves two stages: the first is the definition of the context of the user and the second is the adaptation of the search through considering the user context while selecting relevant information related to the user query. 
The personalization in the field of information retrieval relates to the integration of the user profile in one of the following phases: query reformulation, calculation of the relevance score of the information or presentation of the search results. A user profile can be perceived in the search for information as the main interests and preferences of the user. As for the query, it is discerned as the expression of a need of a user, which will be treated by considering his profile. Several authors have exploited the user profiles for the implementation of the personalization.

It is evident that contextual information is important in any personalization system. Recently, contextual information has been employed as a core element, by the research community, in Web recommendation and personalization systems. That is why it is important to know that integrating relevant contextual elements is crucial in personalized systems. Therefore, we have to take this information into consideration in order to enrich user profiles and then to improve the quality of search results. In fact, as the reach and amount of information on the Web increases, so does the demand for users of tailored services. Thus, Web personalization systems must provide users with not only recommendations for the relevant elements, but also suggest those recommendations in the right situation or context [32].

To be able to personalize the contents for the benefit of a user, it is necessary to know him first. Indeed, if no information is disposed on a user, the contents provided to him will be obligatorily the same for the different users. Therefore, it is essential to know the user better through the establishment of a user model. This model is considered as the heart of all personalization systems. The purpose is to represent the user of a system and personalize the content, the presentation and the navigation. According to Kostadinov [23], the user model is a source of knowledge and a database of a user. Indeed, it represents a particular user or group of users by persistent data containing several characteristics, in the form of profiles. A user template contains a set of information; some are directly related to the user, while others are related to its context. According to [38], these characteristics are as follows: Preferences, Areas of Interest, Tasks and Goals, Experiences, Knowledge and Skills, Personal Data.

User profiles are unique pages where user can express his thoughts and feelings, post photos and show his network of friends [42]. In fact, personalized recommendation systems give recommendations based on user-specific information, so it is essential to give importance to the representation and the construction of the user profiles. In the field of user knowledge representation, several models of ontology-based users have appeared in the literature. We have proposed a user model in a previous work [26].

The fundamental purpose of any context-specific personalization system is to provide proactive services that consider the context of the users. If the contextual behavior of the system is opposed to their understanding and responds differently to user expectations, the success rate of applications drops dramatically. Therefore, personalization is essential for the success of these systems [22].

Some contextual personalization systems use ontological user models like Hawalah [15], Gupta [12], Essayeh [8], Zhang [41], Heckmann [16], others use the user model in ontology-based personalized recommendation systems such as Brut [6], Kadima [21], Su [37], Hudli [17], Ameen [1], and Rattanasawad [34]. 


\section{Ontological User Model for Personalized Search}

In this section, we present some concepts of the proposed ontology. Then, we show enrichment with some inference rules in a first place and in a second place with concepts of FOAF ontology.

\subsection{Ontological Model Concepts}

After collecting information about handicraft women, we need to model them in a structured manner. In order to represent this information, we need to use a model that is performing and efficient for manipulating knowledge and inferencing new facts. For that, we use ontological modeling. Main concepts are extracted from already established and preprocessed interviews. Interviewed women are actually representative of the handicraft women population thanks to the diversity of their activities, their ages, their intellectual levels, etc., and their location at diverse cities in Tunisia and Algeria. To create an ontology, we followed few steps that are necessary to have an accurate and validated ontology. There are different methodologies to create an ontology. We followed the Methontology methodology proposed by Fernandez [9] and Grüninger [11] which primarily considers three steps that are Conceptualization, Formalization and Validation. Conceptualization requires the ontology objective definition and the definition of its concepts, relationships and constraints. The formalization consists in expressing the ontology in a formal language in a specific tool. Finally, the validation is performed by the instantiation of ontology with actual instances on the user.

Before presenting the proposed ontology model, we collected all the necessary concepts. Initially, we begin with creating ontology in [26]. In this ontology, we defined concepts related to domain ontology (which are customer, supplier, handicraft woman, raw material, production tools and products.) and concepts related to user model. Concepts related to user profile are skills, capability, preferences, interest and personal information. Concepts related to the context are User Context (Activity, ICT_use and Intellectual_level), Computing Context (Device) and Physical Context (Location, Time and Environment). We have only described domain concepts and user model concepts without social network concepts. Then we enriched this ontology by SWRL rules for personalization purposes.

In the first proposed ontology, in [26], we adopted that the concepts context and profile are disjoint but it reveals that these two concepts are close to each other and one may be subclass of the other. In our case, we opted for the context as the upper class and the profile is the subclass of it. In fact context is the upper concept of three concepts, in our work, that forms the set of contextual dimension. These concepts are Profile, Environment and Platform. The profile gives information about the user so we defined concepts that may describe the user profile in a personalization system like Personal information, Interests, Preferences, Skills and Abilities.

Fig. 1 presents a fragment of our ontology that contains main classes of our user model ontology.

- Actor: Describes a handicraft woman and the people with whom she has a relationship (supplier and customer). 
- Context: Describes the contextual dimension of handicraft woman

- Platform: Describes information about used platform which is divided into Hardware and Software.

- Environment: Describes information about environment like Location and Time.

- Location: Describes information about location which is divided into Country and City.

- Profile: Represents user profile

- Personal information: Represents personal information such as date of birth, address, marital status.

- Competence: Contains the data that define the skills of handicraft woman in a particular area, such as experience in the domain.

- Interests: Describes information about interests

- Ability: Describes information about user's abilities.

- Preferences: Represents the preferences of handicraft woman. It is divided into General preferences and Application related preferences.

- Language: Describes information about the spoken language.

- Experience: Describes information about experience.

- Job: Describes information about user job.

- Intellectual_level: describes information about user intellectual level.

- ICT_use: describes information about the user ability to use ICT.

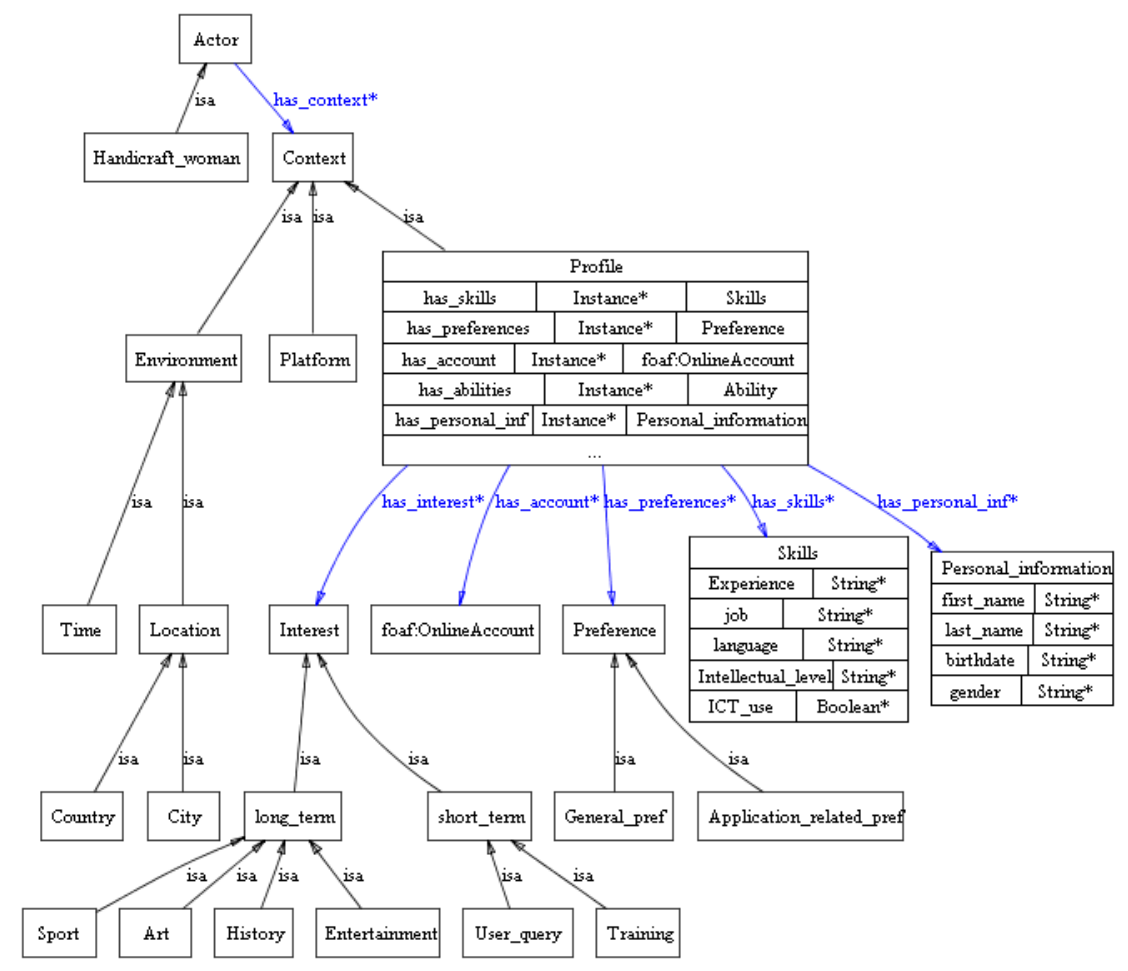

Fig. 1. A fragment of our ontology. 


\subsection{Ontology Enrichment}

Knowledge inference is the fact to use information provided explicitly to infer new knowledge. In order to do this, we use SWRL rules language to classify users into categories. The descriptive logic rules can be:

- Deductive rules: allow to deduce a fact from a set of conditions.

- Reactive rules: allow to execute an action if a set of conditions are verified.

\subsubsection{Deductive SWRL rules for the classification purpose}

Enrichment by SWRL rules is proposed in order to classify handicraft women into categories and to personalize search results according to their category. We noticed that the main different characteristics that we can be based on to do this classification are intellectual level, ICT use and experience. Thus, we define the first set of rules that permit to designate if a handicraft woman is illiterate, has primary school level, has secondary school level or has university level. The second classification shows two types of handicraft women: those using ICT technology and those who do not use it. The third classification leads to classify handicraft women according to their experience. She may be whether beginner or expert in her work. Some SWRL rules are described in previous work [26] so as to personalize query results sent by her through our system.

The first criterion is based on the handicraft women intellectual level. These are our SWRL rules of classification according to the first criterion:

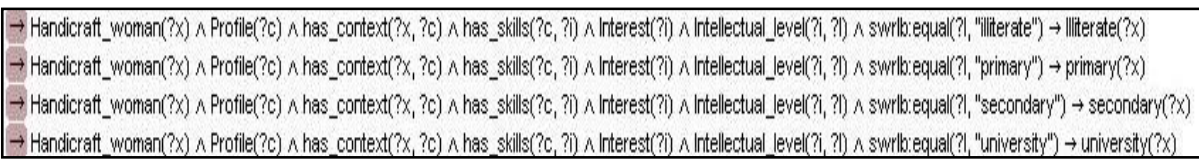

The second criterion is based on ICT use which means that some women use internet and ICT technology and others don't. These are our SWRL rules used for classification according to the second criterion:

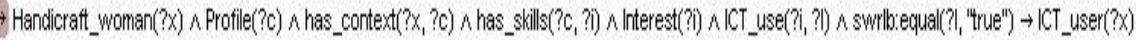

$\rightarrow$ Handicraft woman(?x) A Profile(?c) A has context(?x,?c) A has skills(?c,?i) A Interest(?i) A ICT use(?i, ?) A swrlbrequal(?), "false") $\rightarrow$ Not _CT user(?x)

The third criterion is experience. If women have less than 4 years of work in this job so they are beginners otherwise they are experts in this domain. These are our SWRL rules used for classification according to the third criterion:

$\rightarrow$ Handicraft_woman(?x) A Profile(?c) A has_context(?x,?c) A has_skills(?c, ?i) A Interest(?i) A Experience(?i, ?1) A swrlbrgreaterThan(?!, "4") $\rightarrow$ Expert(?x)

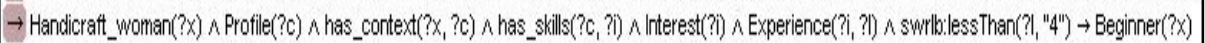

\subsubsection{Reactive SWRL rules for personalization purpose}

In order to recommend a given service, we have to know the experience of the handicraft woman. For instance, if she is beginner we can propose to her Basic training containing the basic notion of the technical knowledge concerning a chosen 
business. Then if she is expert we can propose to her an Advanced Training containing more detailed information about her business (she would have a bigger experience than the first one and she would have an ability to learn detailed notions or new available techniques).

- Handicraft woman $\wedge$ beginner $\rightarrow$ propose basic training

- Handicraft woman $\wedge$ expert $\rightarrow$ propose advanced training

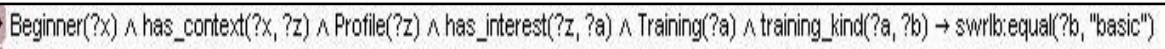
$\rightarrow$ Expert(?x) $\wedge$ has_context(?x, ?z) $\wedge$ Profile(?z) $\wedge$ has interest(?z, ?a) $\wedge$ Training(?a) $\wedge$ training_kind(?a, ?b) $\rightarrow$ swowbrequal(?b, "advanced")

The use of new technologies can facilitate the life and also the job of a handicraft woman. For that, if we want to improve the socio-economic level of the handicraft woman, we should know whether she uses ICT or not. If she uses these new technologies, we can propose her, for example, a training in French on the internet.

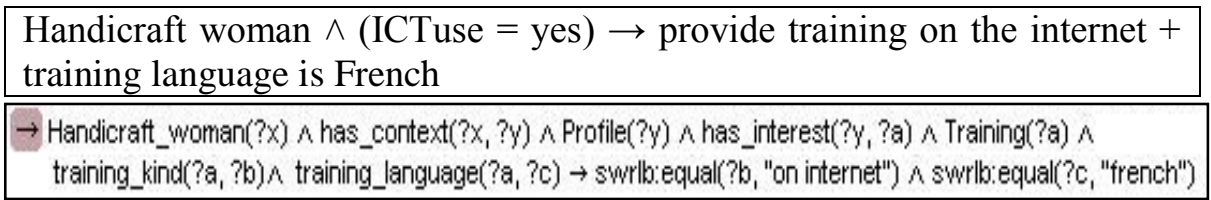

Web content recommendations are based on the user's browsing history. In fact, when the user enters his search query, some search results provided as a response are stored in the user profile model and are then used to derive the recommended links.

This rule infers certain links to the user based on previous search results. In fact, the query terms (keywords) used by a user in his searches reflect his short-term interests. That is why, in our ontology, the concept of "short_term_interest" is composed of a couple of concepts: the "user_query" concept and the "search_results" concept. These concepts are dynamically supplied by the application. According to this, each time the user enters his query, the keywords and the results of the query are stored in the ontology.

SWRL Rule

Handicraft_woman(?x) A has_context(?x, ?y) A Profile(?y) A has_interest(?y, ?z) A User_query(?z) A has_sresults(?z, ?v)

A Search_results(?v) A title(?v, ?t) $A$ url_s(?v, ?u) A has_recommendation(? $\left.x_{1} ? \mathrm{~h}\right)$ A Recommended_links(?h) A

url_r(?h, ?s) $\rightarrow$ url_r(?h, ?u)

We define three recommendation rules for recommending articles to the user with similar context with the contexts of the users in our ontology. These rules concern production tools and raw materials as elements to recommend. We choose these items as the area of our work is in the domain of handicraft. These rules are presented in previous works [28].

\subsection{Enrichment by FOAF Concepts}

Our system permits us to personalize search results based on information about social network profile of the handicraft women. Thus, we need a structure to save this important information. FOAF ontology [5] is known as an ontology that represents persons (foaf:person) and their relations between them (foaf:knows). It contains 
concepts that we need in order to get information about a person, his relationship with others and his online account (which can be a social network account) such as foaf:person, foaf:online_account and foaf:project which can describe information about handicraft woman, her social network profile and her products respectively. Thus, we reused some concepts from FOAF ontology. We have already proposed in our previous work [27] an enrichment of our initial ontology with FOAF concepts with bridge axioms and semantic relationships.

The main concepts that we need from this ontology to describe a handicraft woman profile or any other actor profile in his presence in social network are: foaf:Person, foaf:online_account and the relationships related to these concepts are foaf:knows, foaf:has_account.

\section{$5 \quad$ Integrating Ontology in an Interactive Information Retrieval System}

We propose an approach, which is illustrated in Fig.2, in order to create an ontology for user model in handicraft domain and in order to assist handicraft woman in their search in social network. This approach is composed of three steps which are: the first is the extraction and elicitation from interviews with handicraft women from Tunisia and Algeria. The second step is ontological modeling of extracted knowledge. The third and the last step is the integration of the ontology in an interactive system.

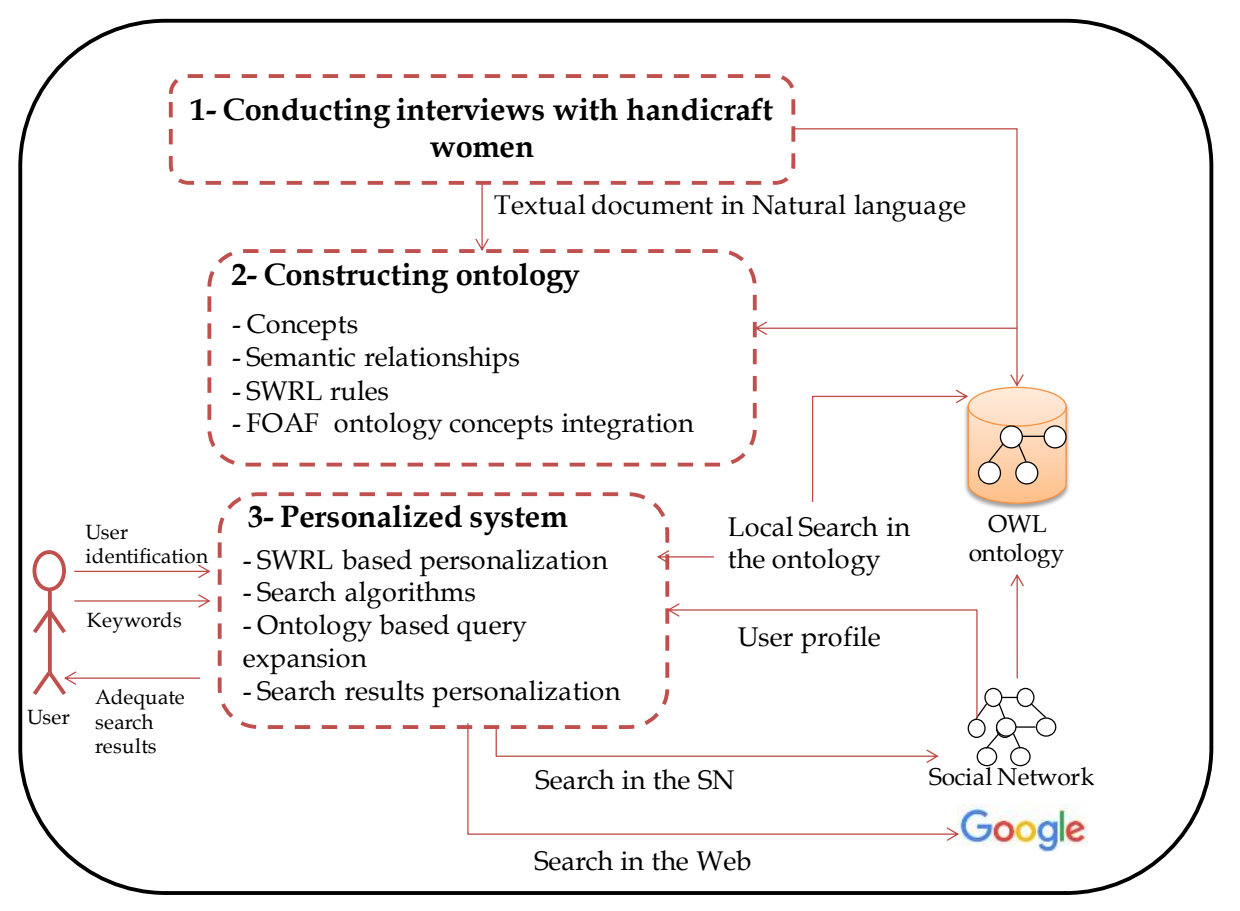

Fig. 2. Ontology-based information retrieval system architecture. 


\subsection{Conducting Interviews with Handicraft Women}

In this step, we collect information about handicraft women. This is established through interviews that we made with them in many areas of Tunisia and Algeria. At this stage, we got a set of interviews in textual form. We preprocessed these interviews through many manual operations to eliminate ambiguity and repetition. Then, we extracted the most useful information related to the domain, the personal information and the ICT use. It mainly concerns the name, the age, etc. as personal information, the production tools, the raw materials, etc. as information about their domain and their readiness to use new technologies, their interests, etc. as information about ICT use.

\subsection{Constructing Ontology}

Acquiring information about users in a personalized system is a crucial task. It can be explicitly or implicitly acquired for personalizing search results. Apart from interviews, social network user profiles have been used to implicitly extract information about users' interests for personalization. For instance, Facebook user profiles which contains personal and contextual information such as gender, date of birth, language, location, etc. permit to implicitly extract user information from his social network account. This information is saved by the majority of users in their profiles and can be extracted. This helps us to find better results in information retrieval.

In this step, we create the ontology already described in section 4 . Then, the knowledge extracted, implicitly from Facebook user profiles and explicitly from data provided by the user through the form interface, is then used to instantiate the ontology. In fact, ontology is not only an effective mean of modeling digital information and user context, but also, it can be a very useful tool for improving navigation effectiveness as well as personalized search results and query refinement because it represents an overview of the domain, related to a specific area of interest. The information gathered about the user and represented by our ontology, will be instances of concepts in the ontological user model.

\subsection{Personalized System}

We create two search engine categories that focus on different sites. Once we have defined our search engines, they can be accessed through a search box in our web application to help users make searches from our web application. At the first place, the user has to choose the search modality he wants to use. He has the choice to search either on "Facebook" or on the integrity of the Web "Google". After the selection of search type, the user will be able to input his query. For the local search in the ontology, it concerns the description of some tools or materials related to handicraft domain.

In this step, first the user enters the search word. Then, after collecting data related to the user in the previous step, the user selects the information that can help him to improve search results and uses them in search. The selection focuses on the information and data that are appropriate to seek the word. Then, search results are 
provided to the user in a personalized way in order to fit his needs and preferences. If a user selects refinement labels, or categories of search results, we append his queries with additional search terms to help him retrieve more targeted results or redirect him to a different page, so that he can refine his searches and get quick answers.

\subsubsection{Search on integrity of the web module}

If the user selects search on integrity of the web, for the search results and query personalization, we used the Google Custom Search Control API that enables us to create a Custom Search Engine (CSE) and then to embed the resulted custom search element in our web application. Moreover, custom search enables us to append search terms to our users' queries (rewrite queries) and this is possible to be implemented through refinements labels in order to get relevant searches and quick answers. After that, we attributed weights to the inclusive labels. This is helpful to promote or demote a tagged site so that to adjust the ranking of the search results provided by our search engine. Search on integrity of the web is well detailed in a previous work [35].

\subsubsection{Query expansion module}

There are several models and algorithms which are used in the search for information. We rely on the query reformulation model. This model is necessary to target the search for relevant documents by increasing the query terms got from the user profile. Within this model, there are several algorithms used. Among them, we use Rocchio algorithm in our personalized search on Facebook. Search based on this algorithm identifies the user profile as a pair of concepts from an ontology and operates in the query reformulation. The first concept represents the relevant concepts from the user and the second concept represents the irrelevant concepts selected. In our approach we use the same principle of Rocchio algorithm in the query reformulation using terms from the user profile. We proposed an extension of the formula used in this algorithm. We identify the user profile as being a pair of concepts through an ontology. The first concept is the profile which is composed of personal data and the second concept is the context which is composed of context data. The query reformulation is made by applying the equation as follows:

$$
\mathrm{Q}_{2}=\alpha \mathrm{Q}_{1}+\beta \operatorname{Prf}+\gamma \text { Cont. }
$$

In this formula, Q1 represents the original query, Q2 represents the new query, Prf is a vector of terms representing the concept of profile and Cont is a vector of terms representing the concept of user context. The factors $\alpha, \beta, \gamma$ are weights associated with, respectively, the original query, profile concept and the concept context such as:

$\alpha+\beta+\gamma=1$ and $\alpha=\beta+\gamma$.

\subsubsection{Search on Facebook module}

If the user selects search on Facebook, the search results will be displayed according to the chosen type. In this step, the user must select the type of results he prefers. The result type must be either page or group or user. This step determines the type of 
results displayed to the user adapted to its needs. We added a third type of search based on contextual and personal information (stored in our ontology) to reformulate the query and to ameliorate the search results. The purpose of this module is to obtain different types of results with the use of profile and context of the user to improve the search. To retrieve information from user context, we extract information that characterizes the context namely location and time. Then, we apply search by selected type of search and using the profile and contextual information extracted in the previous step. We choose the profile information and user context information that has a relationship with the personalized search to improve performance and to meet the user needs.

\subsubsection{Personalized recommendation module}

In this step, we recommend an item to the user after comparing the contexts. In fact, when the user performs a search on the social network via our application he must be connected to his account on the social network. The profile and context of the user are saved in our ontology. In order to recommend elements of the ontology we compare the context of the user with other contexts of other users in the ontology. If they have similar contexts, they probably need the same raw materials, for example. Otherwise, we do not recommend items for him. This recommendation is based on the SWRL rules that we have previously defined.

\section{$6 \quad$ Results and Discussion}

\subsection{Results}

Personalization is a process that adjusts the ranking system based on context-specific user discovery. We will compare the accuracy of the search results returned before and after personalization of a user who chooses the search category in "Web Integrity". He submits as a search term "pottery".

We notice that the first search results delivered to the user are general results and can be returned to anyone entering the same query. On the other hand, when he executes different refinement labels, the search results are then more specific to what he is looking for and customized to meet his needs. For example, by clicking on the "clay" refinement label, we add the search terms "ceramic" and "clay" to the original query of the user. As a result, the returned search results are then more relevant and match his needs.

For the first type of search, on the integrity of the web, we can conclude that the results given after personalization are better than those given at the beginning for the reason that they increase, as we demonstrated in [35], user satisfaction with a short response time.

For the second type of search, on Facebook, the first results given for our research concern the status. The contents of the returned status, related to the search of the user, are written in other languages that are not understandable and that are not relevant to the user. For this, we added a step that allows the user to select the type of result. The results types are either "pages", "groups" or "users", but the returned 
results are not yet adapted to the needs and preferences of the user. To improve our search, we used the account information in which we find information about the user's profile and context. The user, when he has done his research, can choose the type of result and can add labels that represent his interests and preferences.

In this case, we obtain results that are better adapted to user needs and remarkably improved. According to the obtained results, we perceive that the search has become more focused and provides more relevant results. The search for information in the statutes is very expensive in terms of time consumed to produce results; it can even last infinity.

For the second type of search, the user can obtain information in the form of pages, groups or users. The search time for the information search is four and a half seconds. For the latter type, which is based on the use of the profile and the context, the search results become more relevant to the needs of the users and the execution time of this type of search is one second. These results are shown in Fig.3.

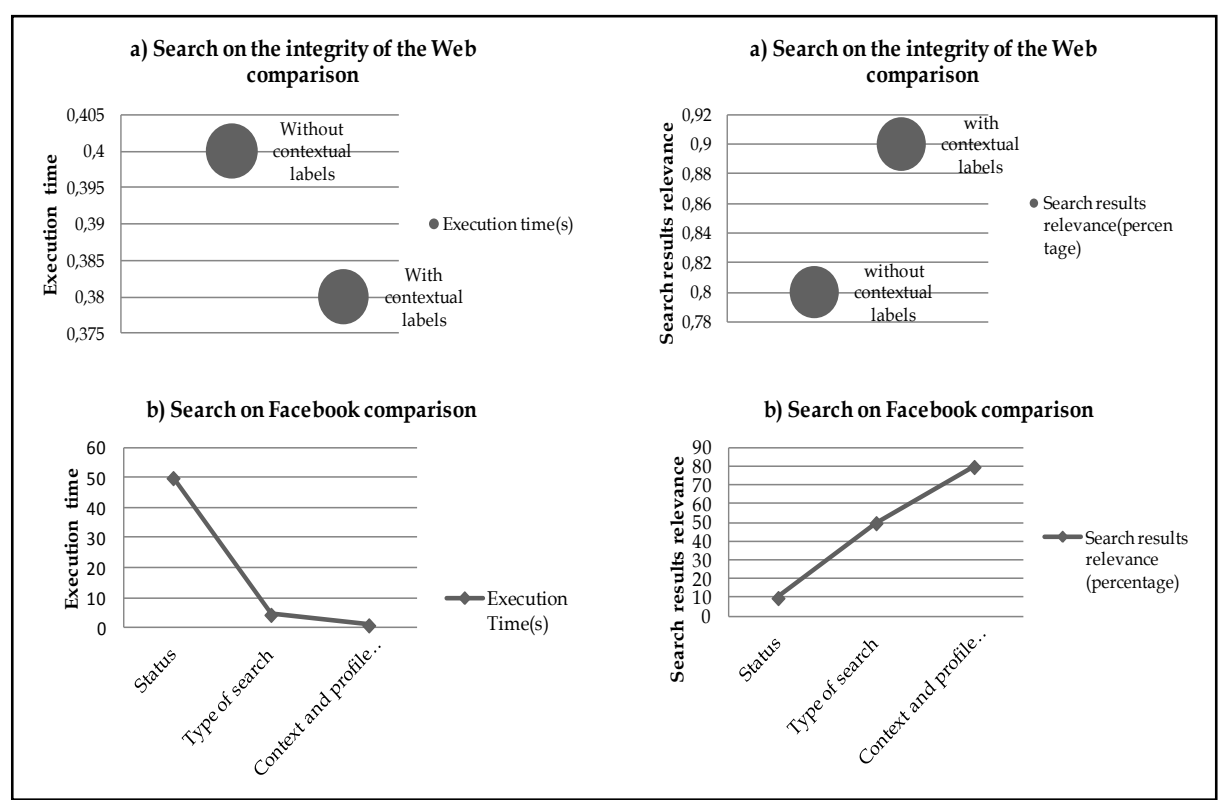

Fig. 3. Search on the integrity of the web comparison and search on Facebook comparison.

\subsection{Discussion}

The proposed model has a generic part that is related to the user model. In fact, the concepts defined to describe the context are applicable for any user in any domain as same as for the user profile. The specific part of the model concerns the field of application. In fact, the model can be used for other domain if the part related to the domain is changed by the expert in order to model concepts for the targeted domain.

The defined rules are two folds. Some are specifically meant to classify users according to some characteristics. Those rules are generic and can be applied in any 
field where the objective is to classify users for some personalization purposes. Other rules, which are related to recommendation, are specific to the domain (handicraft domain). Therefore, if the expert wants to reuse these rules, he has to extend the model by concepts of his field of application.

The concepts chosen to describe the context part are mainly inspired by the work of Bacha [2] and which are user, environment and device. This representation of the context notion is suitable so as to determine, which are the main elements that can describe and indicate the interaction context between a user and a machine. At the interaction time, the user profile, the device employed and the time and location of the interaction can affect the experience of using a personalization system.

The choice behind using ontology to represent the user model is explained by the ability of this technology to take into account the semantic relationship between concepts and the semantic of the concepts themselves. However, the use of this technology is not limited and can be expanding by using other models like UML (Unified Modeling Language) diagrams in order to describe dynamic diagrams and functional diagrams.

The proposed user model could also be represented with other models (e.g. UML) and the recommendation could also be done with traditional techniques of data mining (e.g. association rules). This paper confirms that Semantic Web Technologies can be used for the same purpose but it is more interesting to use them in order to preserve the semantic in the model.

Ontologies make it possible to better represent and keep the semantics of the concepts represented and their relationships. Their construction and use in personalization was not always obvious due to the complexity of the domains to be represented and the non-limitation of the number of concepts to be created to better cope with the personalization thereafter.

The advantage of using our ontological model is that it is extensible at any moment, that is to say, we can add concepts that are supposed to be necessary for a given objective.

The modification of the names of the concepts or the suppression of some concepts is not conceivable or advisable because this will affect the SPARQL queries developed in the ontology.

The model of the proposed context meets the criteria of personalization because it makes it possible to detect the profile of the user and to enrich his request. The change of context must be detected automatically without the intervention of a human being.

In our study we wanted to focus on the inference side of ontology using the SWRL rules in its two forms; deductive for the classification of individuals and reactive for the personalization.

The good side of using these rules is that they allow reasoning on explicit data and infer the necessary personalization related to these data.

The bad side is that the complexity is exponential with the expansion of the knowledge base i.e. the ontology becomes cumbersome and consumes more resources and time for the execution of the SWRL rules.

The results show that working with ontology based models proves that it is more efficient and easier to achieve than with other models. 


\section{Conclusion}

In this paper, we proposed an approach that highlights the role of the proposed ontology in the content personalization. This ontology is built as part of a case study of handicraft domain. In this context, we have implemented the proposed approach for personalization through the integration of our ontology in a personalization system. Our ontology serves as a bridge to deliver personalized content to handicraft women based on their profiles. In addition, we have proceeded to classify these users into categories through SWRL rules. Thus, the information characterizing the user and SWRL rules are implemented to be exploited to enrich the user queries. Indeed, this method enriches the user query by a set of predicates contained in their profile. Therefore, they allow better tailoring responses to users according to their profiles. For the search results and query personalization, we have defined two search engine categories that covered the modality of search on Facebook and on the integrity of Web. The result of our approach is encouraging. In fact, we have relied on SWRL rules language to improve query results and therefore to better meet user needs. Moreover, custom search has enabled us to append search terms to our users' queries (rewrite queries) and this has been possible to be implemented through refinements labels in order to get relevant searches and quick answers.

In our future works, we aim at adding other contextual and profile information describing a user. This extension will provide users with more personalized results. Second, we look at testing our prototype and our approach to other social networking sites. We aim, furthermore, at formalizing more our ontology by proposing other SWRL rules. In fact, those rules help us to infer new knowledge, from the user profile model, which is useful to enhance handicraft woman's search experience and satisfy her with more relevant personalized results. Another interesting prospect is to implement our contribution in the interactive system of the project. The idea to test and evaluate the use of our approach to other fields is one of our future works.

Acknowledgements. We are very thankful to the Algerian Tunisian Project dealing with the improvement of handicraft women business in emerging countries through affordable technologies and social networks.

\section{References}

1. Ameen, A., Khan, K.U.R., Rani, B.P.: SemRPer - A Rule based Personalization System for Semantic Web. International Journal Web Applications, Volume 7, Issue 1, March, 2015, pp. 23-38 (2015)

2. Bacha, F., Oliveira, K., Abed, M.: Using context modeling and domain ontology in the design of personalized user interface. International Journal on Computer Science and Information Systems (IJCSIS), vol. 6, pp. 69-94 (2011)

3. Breslin, J.G., Harth, A., Bojars, U., Decker, S.: Towards Semantically-Interlinked Online Communities. In: Proceedings of the Second European Semantic Web Conference, ESWC 2005, May 29-June 1, 2005, Heraklion, Crete, Greece (2005)

4. Brézillon, P., Marie Curie, P.: A context approach of social networks. In: proceedings of the Workshop on Modeling and Retrieval of Context (2004) 
5. Brickley, D., Miller, L.: FOAF Vocabulary Specification. Namespace Document 2 Sept 2004, FOAF Project. http://xmlns.com/foaf/0.1/ (2004)

6. Brut, M., Sèdes, F.: Modélisation basée sur ontologies pour développer des recommandations personnalisées dans les systèmes hypermédia adaptatives. In: 28th congrès INFormatique des ORganisations et Systèmes d'Information et de Décision (INFORSID) 25 - 28 may, Marseille, France, p. 61-76 (2010)

7. Harary, F., Norman, R.Z., Cartwright, D.: Structural Models: An Introduction to the Theory of Directed Graphs. New York: Wiley (1965)

8. Essayeh, A., Abed, M.: An ontology-based framework for enhancing personalized content and retrieval information. In: proceedings of 11th International Conference on Research Challenges in Information Science (RCIS) (2017)

9. Fernández, M., Gómez-Pérez, A., Juristo, N.: METHONTOLOGY: From Ontological Art Towards Ontological Engineering. In: Symposium on Ontological Engineering of AAAI. Stanford (California). March (1997)

10. Ghita, S., Nejdl, W., Paiu, R.: Semantically Rich Recommendations in Social Networks for Sharing, Exchanging and Ranking Semantic Context. In: ESWC Workshop on Ontologies in P2P Communities (2005)

11. Grüninger, M., Fox, M.S.: The Role of Competency Questions in Enterprise Engineering. In: Rolstadås A. (eds) Benchmarking - Theory and Practice. IFIP Advances in Information and Communication Technology. Springer, Boston, MA, pp. 22-31 (1995)

12. Gupta, K. Arora, A.: Web Search Personalization Using Ontological User Profiles. In: proceedings of the Second International Conference on Soft Computing for Problem Solving (SocProS 2012), December 28-30, 2012, Springer, p. 849-855 (2014)

13. Gruber, T.R.: A translation approach to portable ontologies. Knowledge Acquisition 5(2):199-220 (1993)

14. Hardy, R., Rukzio, E., Holleis, P., Wagner, M.: MyState: Sharing Social and Contextual Information through Touch Interactions with Tagged Objects. In: Proceeding MobileHCI '11 Proceedings of the 13th International Conference on Human Computer Interaction with Mobile Devices and Services, p. 475-484 (2011)

15. Hawalah, A., Fasli, M.: Utilizing contextual ontological user profiles for personalized recommendations. Expert Systems with Applications 41(10), pp. 4777-4797 (2014)

16. Heckmann, D., Schwarzkopf, E., Mori, J., Dengler, D., Kröner, A.: The user model and context ontology GUMO revisited for future web 2.0 extensions. In: proceedings of the third International Workshop on Contexts and Ontologies: Representation and Reasoning (C\&O: RR- 2007) at CONTEXT 07, Roskilde Université, Danemark (2007)

17. Hudli, S., Arvind, H.: Learning in Rule-based Recommendation Systems. In: the 27th Annual IEEE Software Technology Conference Long Beach, California, USA 12-15 October (2015)

18. Ingwersen, P., Jarvelin, K.: The Turn: Integration of information seeking and information retrieval in context. Springer (2005)

19. Johansson, F.: Extending Mobile Social Software with Contextual Information. Umeå University, Umeå, Sweden, http://www.signar.se/blog/wp-content/extending-mobilesocial-software-with-contextual-information.pdf (2008)

20. Joly, A., Maret, P., Daigremont, J.: Context-Awareness, the Missing Block of Social Networking. International Journal of Computer Science and Applications 4(2):5065 (2009)

21. Kadima, H., Malek, M.: Toward ontology-based personalization of a recommender system in social network. In: International Conference of Soft Computing and Pattern Recognition (SoCPaR), IEEE, pp. 119-122 (2010)

22. Kawsar, F., Fujinami, K., Pirttikangas, S., Nakajima, T.: Personalization and context aware services: A middleware perspective. In: Proceedings of the 2nd International Workshop on 
Maha Maalej, Achraf Mtibaa, Faïez Gargouri

Personalized Context Modeling and Management for UbiComp Applications, California, USA, vol. 197 (2006)

23. Kostadinov, D.: Personnalisation de l'information et gestion des profils utilisateurs. Rapport de DEA, Université de Versailles, France (2003)

24. Li, J., Wang, H., Khan, S.U.: A Semantics-based Approach to Large-Scale Mobile Social Networking. Mobile Networks and Applications 17(2), pp. 192-205 (2012)

25. Maalej, M., Mtibaa, A., Gargouri, F.: Ontology-Based Context-Aware Social Networks. In: Proceeding of (OAIS) in (ADBIS'2013), September 1-4, 2013, Genoa, Italy, pp. 287295. B. Springer International Publishing Switzerland (2013)

26. Maalej, M., Mtibaa, A., Gargouri, F.: Ontology-based user modeling for handicraft woman recommendation. In: 4th International Conference on Model \& Data Engineering (MEDI'2014) Larnaca, Cyprus, Lecture Notes in Computer Science, Volume 8748, pp. 138-145 (2014)

27. Maalej, M., Mtibaa, A., Gargouri, F.: Enriching user model ontology for handicraft domain by FOAF. In: proceedings 14th IEEE/ACIS International Conference on Computer and Information Science (ICIS), IEEE, pp. 651-655 (2015)

28. Maalej, M., Mtibaa, A., Gargouri, F.: Context Similarity Measure for Knowledge-based Recommendation System Case Study: Handicraft Domain. In: The 14th International Conference on Cooperative Design, Visualization and Engineering, pp. 77-84, Mallorca, Spain (2017)

29. Micarelli, A., Sciarrone, F.: Anatomy and empirical evaluation of an adaptive web-based information filtering system. User Modeling and User-Adapted Interaction, 14(2-3), pp. 159-200 (2004)

30. Moreno, J.: Les Fondements de la sociométrie. PUF, original version (1993)

31. Narayanan, A., Shmatikov, V.: De-anonymizing Social Networks. In: IEEE Symposium on Security and Privacy, pp. 173-187 (2009)

32. Prahalad, C.K.: Beyond CRM: CK Prahalad predicts customer context is the next big thing. American Management Association MwWorld (2004)

33. Qiao, X., Li, X., Su, Z., Cao, D.: A Context-Awareness Dynamic Friend Recommendation Approach for Mobile Social Network Users. International Journal of Advanced Intelligence 3(2), pp. 155-172 (2011)

34. Rattanasawad, T., Buranarach, M., Thein, Y.M., Supnithi, T., Saikaew, K.R.: Design and Implementation of a Rule-based Recommender Application Framework for the Semantic Web Data. In: The First Workshop on Practical Application of Ontology for Semantic Data Engineering (PAOS2013), Seoul, Korea (2013)

35. Rekik, E., Maalej, M., Mtibaa, A., Gargouri, F.: Ontology-based approach to provide personalized search results for handicraft woman. In: 15th International Conference on Intelligent Systems Design and Applications (ISDA), IEEE, pp. 604-609, Marrakesh, Morocco, IEEE Conference Publications (2015)

36. Scott, J.: Social Network Analysis: A Handbook. 2nd. Thousand Oaks, CA: Sage Publications (2000

37. Su, Z., Yan, J., Ling, H., Chen, H.: Research on personalized recommendation algorithm based on ontological user interest model. Journal of Computational Information Systems 8(1), pp. 169-181 (2012)

38. Van Setten, M.: Personalized Information Systems. Giga CE project part of Gigaport Project, Telematica Institut, Netherlands (2001)

39. Wang, X.H., Zhang, D.Q., Gu, T., Pung, H.K.: Ontology based context modeling and reasoning using OWL. In: proceedings of the second IEEE Annual Conference on Pervasive Computing and Communications Workshops, pp. 18-22, Orlando, FL, USA (2004) 
40. White, R.W., Bailey, P., Chen, L.: Predicting User Interests from Contextual Information. In: proceedings of the $32^{\text {nd }}$ international ACM SIGIR conference on Research and development in information retrieval, pp. 363-370, New York, USA (2009)

41. Zhang, Y., Gou, L., Zhou, T., Lin, D., Zheng, J., Li, Y., Li, J.: An ontology-based approach to patient follow-up assessment for continuous and personalized chronic disease management. Biomedical Informatics, vol.72, pp. 45-59 (2017)

42. Zhou, X., Xu, Y., Li, Y., Josang, A., Cox, C.: The state-of-the-art in personalized recommender systems for social networking. Artificial Intelligence Review 37(2), pp. 119-132 (2012)

43. Zigkolis, C., Kompatsiaris, Y., Vakali, A.: Information analysis in mobile social networks for added-value services. In: proceedings of the W3C Workshop on the Future of Social Networking, Barcelona, Spain (2009)

44. Zitnik, S.: Collective Ontology-based Information. In: proceedings of the 24th International Conference on Advanced Information Systems Engineering CAiSE'12. Gdańsk, Poland (2012) 
Maha Maalej, Achraf Mtibaa, Faïez Gargouri 